Typeset with jpsj2.cls <ver.1.2.2 $>$

Full PAPeR

\title{
Three-Dimensional Multiband d-p Model of Superconductivity in Spin-Chain Ladder Cuprate
}

\author{
Shigeru Koikegami * and Takashi Yanagisawa ${ }^{1}$ \\ Second Lab, LLC, 2-32-1 Umezono, Tsukuba, Ibaraki 305-0045 \\ ${ }^{1}$ Nanoelectronics Research Institute, AIST Tsukuba Central 2, Tsukuba, Ibaraki 305-8568
}

We study the superconductivity in the three-dimensional multiband d-p model, in which a $\mathrm{Cu}_{2} \mathrm{O}_{3}$-ladder layer and a $\mathrm{CuO}_{2}$-chain layer are alternately stacked, as a model of the superconducting spin-chain ladder cuprate. $p_{z}$-Wave-like triplet superconductivity is found to be the most stable, and its dependence on interlayer coupling can explain the superconducting transition temperature dependence on pressure in real superconducting spin-chain ladder cuprates. The superconductivity may be enhanced if hole transfer from the chain layer to the ladder layer can be promoted beyond the typical transfer rate.

KEYWORDS: superconductivity, three-dimensional multiband d-p model, spin-chain ladder cuprate, interlayer coupling

\section{Introduction}

The spin-chain ladder cuprate $\mathrm{Sr}_{14-x} \mathrm{Ca}_{x} \mathrm{Cu}_{24} \mathrm{O}_{41}$ (Sr14-24-41) has attracted much interest as a related material of high- $T_{\mathrm{c}}$ cuprates for more than two decades. It has three types of layers, known as the chain, spacer, and ladder. ${ }^{1-3)}$ These layers are stacked in an alternating manner along the layer axis $b$. The chain layer contains $\mathrm{CuO}_{2}$ chains, while the ladder layer consists of two-leg $\mathrm{Cu}_{2} \mathrm{O}_{3}$ ladders. Theoretically, the two-leg spin- $1 / 2$ antiferromagnetic Heisenberg ladder is a system with a finite energy gap for reaching the lowest magnetic excitation. ${ }^{4)}$ The hole-doped two-leg spin ladder has been investigated theoretically and confirmed numerically to possess an instability towards $d$-wave-like superconductivity on the basis of the $t$ - $J$ ladder model, ${ }^{5-10)}$ one-band Hubbard ladder model, ${ }^{10-19)}$ and three-band Hubbard ladder model. ${ }^{10,20)}$ In 1996, $\mathrm{Sr}_{14-x} \mathrm{Ca}_{x} \mathrm{Cu}_{24} \mathrm{O}_{41}$ with $x=13.6$ was found to be superconducting under high pressures, $P \geq 3 \mathrm{GPa} .{ }^{21)}$ In Sr14-24-41, doped holes move from the chain layer to the ladder layer by Ca substitution for Sr. ${ }^{22}$ ) High pressures intensify this self-doping effect and afford the electrons in the ladder layer sufficient itinerancy. ${ }^{23,24)}$ However, the superconducting transition temperature $\left(T_{\mathrm{c}}\right)$ has a maximum at $P \approx 5 \mathrm{GPa}$ and then decreases with increasing pressure. $\left.{ }^{25}\right)$ The dependence of $T_{\mathrm{c}}$ on pressure is common to other compositions as well, such as $\mathrm{Sr}_{14-x} \mathrm{Ca}_{x} \mathrm{Cu}_{24} \mathrm{O}_{41}$ for $x=11.5^{26)}$ and $x=10,11,12 .{ }^{27,28)}$ Such superconductivity behavior is similar to that of high- $T_{\mathrm{c}}$ cuprates in the overdoped regime. Considering the experimental results of the electronic properties, the superconductivity of Sr14-24-41 can be

\footnotetext{
${ }^{*}$ E-mail address : shigeami@secondlab.co.jp
} 
thought of as an extension of that of high- $T_{\mathrm{c}}$ cuprates. ${ }^{29)}$

In accord with the above novel results, the two-leg Hubbard ladders coupled via a weak interladder hopping were investigated theoretically by Kishine and Yonemitsu. ${ }^{30)}$ According to their perturbative renormalization group analysis, the system has a $d$-wave-like superconducting ground state, and restores the interladder coherence within an increase in the extent of interladder hopping. Moreover, the superconducting state and its $T_{\mathrm{c}}$ were evaluated on the basis of the two-dimensional (2D) Trellis-lattice Hubbard model, i.e., the coupled two-leg ladder model. ${ }^{31-33)}$ In their studies, a wide doping regime exists in which $d$-wave like singlet superconductivity appears. In one of them, ${ }^{32)}$ it is also shown that a certain doping regime prefers $p_{z}$-wave like triplet superconductivity. Thus, it is plausible that the superconductivity may be intrinsically linked to the $2 \mathrm{D}$ two-leg ladder structure. Practically, however, the superconductivity in Sr14-24-41 appears for certain compounds only under high pressures. In order to understand this situation, we should find routes for enhancing superconductivity in real compounds, that have not been considered yet in past theoretical studies. It is worth noting that Isobe et al. have already pointed out the effects of the hybrid orbital between $\mathrm{Cu} 3 d$ in the ladder layer and $\mathrm{O} 2 p$ in the chain layer on the superconductivity under high

pressures. ${ }^{25,34)}$ According to their arguments, the hybrid orbital accelerates the redistribution of holes and enhances the superconductivity.

In this study, we investigate the superconductivity of the two-leg ladder layer coupled with the chain layer. We adopt the three-dimensional (3D) d-p model with the quasi-onedimensional (Q1D) structure in which a $\mathrm{Cu}_{2} \mathrm{O}_{3}$ ladder layer and a $\mathrm{CuO}_{2}$ chain layer are alternately stacked. In our model, the ladder and chain layers are coupled via hybridization between the $\mathrm{Cu} 3 d$ orbital in the ladder layer and the $\mathrm{O} 2 p$ orbital in the chain layer. Moreover, we introduce a sufficiently small on-site Coulomb interaction showing that the second-order perturbation theory (SOPT) can be justified. We can treat the superconductivity using a weak coupling analysis because, in our model, the effective interaction for Cooper pairing is so small that only electrons on the Fermi surface are involved in the superconductivity. In particular, the weak coupling formulation by Kondo is applicable even in the case with a very small effective interaction. ${ }^{35)}$ We previously applied Kondo's formulation to the study of the 3D d-p model with multilayer perovskite structure and investigated how the superconducting gap depends on the number of layers. ${ }^{36)}$ As in the study of multilayer cuprates, we show that calculation on the basis of a 3D model is practical for assessing the superconductivity in spinchain ladder cuprate. Our results give a possible explanation as to why the superconductivity appears in $\mathrm{Sr}_{14-x} \mathrm{Ca}_{x} \mathrm{Cu}_{24} \mathrm{O}_{41}$ for $x \geq 10$ only under high pressures.

\section{Formulation}

Our 3D d-p model is schematically shown in Fig. 1. We consider two $\mathrm{Cu}$ sites and three $\mathrm{O}$ sites in the ladder layer, and one $\mathrm{Cu}$ site and two $\mathrm{O}$ sites in the chain layer. In the chain layer, 
(a)

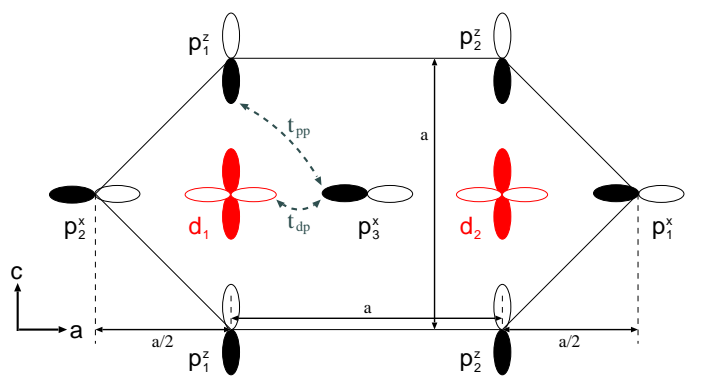

(b)

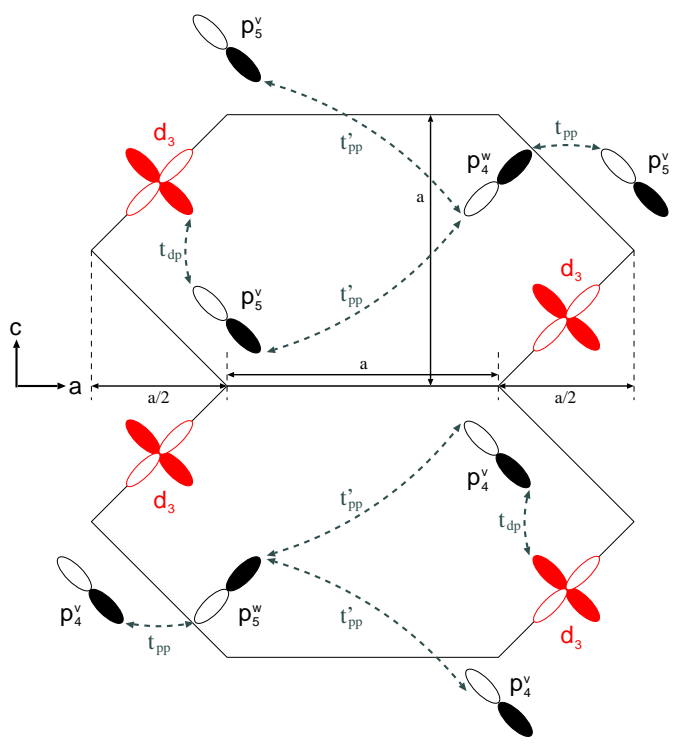

(c)

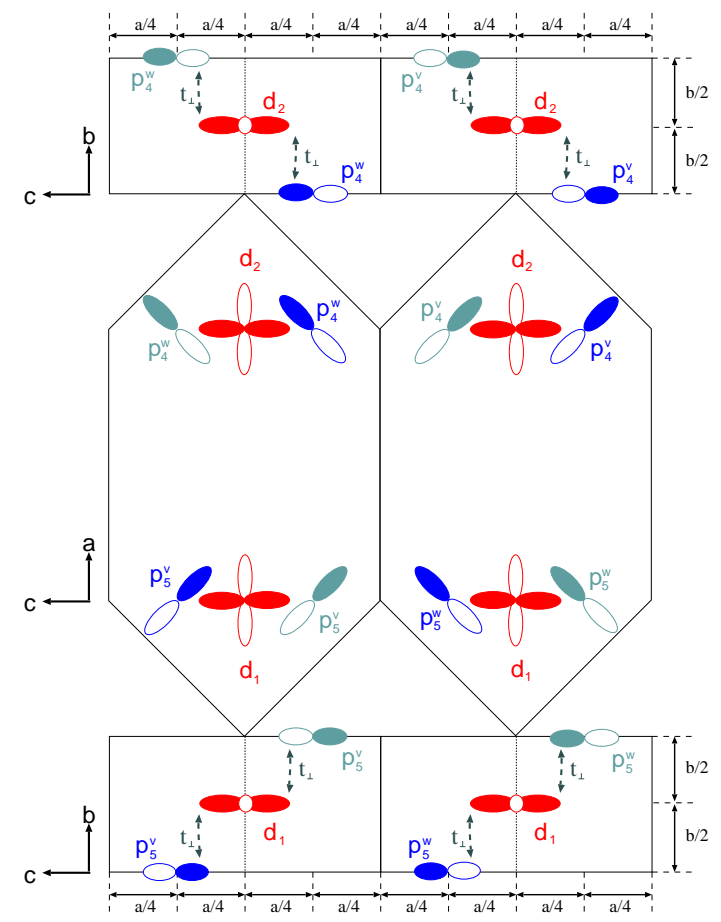

Fig. 1. (Color online) Transfer energies in the 3D chain-ladder d-p model: (a) within the ladder layer, (b) within the chain layer, and (c) for the interlayer hopping. 
J. Phys. Soc. Jpn.

Full PAPER

the lattice constants along the c-axis and a-axis are $\sqrt{2}$ and $1 / \sqrt{2}$ times those of Sr14-24-41, respectively. Owing to this technique, we do not need a large unit cell, which is needed in crystal analysis of Sr14-24-41, in order to treat the interlayer hopping. We can decompose our 3D d-p model composed of the ladder layer and chain layer into several parts as follows:

$$
\begin{aligned}
H=H_{\text {Ladder }}^{0}+ & H_{\text {Chain }}^{0}+H_{\text {Ladder-Chain }}^{0}+H^{\prime} \\
-\mu \sum_{\boldsymbol{k} \sigma}[ & d_{1 \boldsymbol{k} \sigma}^{\dagger} d_{1 \boldsymbol{k} \sigma}+d_{2 \boldsymbol{k} \sigma}^{\dagger} d_{2 \boldsymbol{k} \sigma}+p_{1 \boldsymbol{k} \sigma}^{z \dagger} p_{1 \boldsymbol{k} \sigma}^{z}+p_{1 \boldsymbol{k} \sigma}^{x \dagger} p_{1 \boldsymbol{k} \sigma}^{x}+p_{2 \boldsymbol{k} \sigma}^{z \dagger} p_{2 \boldsymbol{k} \sigma}^{z}+p_{2 \boldsymbol{k} \sigma}^{x \dagger} p_{2 \boldsymbol{k} \sigma}^{x}+p_{3 \boldsymbol{k} \sigma}^{x \dagger} p_{3 \boldsymbol{k} \sigma}^{x} \\
& \left.\quad+d_{3 \boldsymbol{k} \sigma}^{\dagger} d_{3 \boldsymbol{k} \sigma}+p_{4 \boldsymbol{k} \sigma}^{w \dagger} p_{4 \boldsymbol{k} \sigma}^{w}+p_{4 \boldsymbol{k} \sigma}^{v \dagger} p_{4 \boldsymbol{k} \sigma}^{v}+p_{5 \boldsymbol{k} \sigma}^{w \dagger} p_{5 \boldsymbol{k} \sigma}^{w}+p_{5 \boldsymbol{k} \sigma}^{v \dagger} p_{5 \boldsymbol{k} \sigma}^{v}\right],
\end{aligned}
$$

where $d_{l \boldsymbol{k} \sigma}\left(d_{l \boldsymbol{k} \sigma}^{\dagger}\right)$ and $p_{m \boldsymbol{k} \sigma}^{\nu}\left(p_{m \boldsymbol{k} \sigma}^{\nu \dagger}\right)$ are the annihilation (creation) operators for d-electrons in the $l$-th site and for $\mathrm{p}^{\nu}$-electrons in the $m$-th site, having a momentum $\boldsymbol{k}$ and spin $\sigma=\{\uparrow, \downarrow\}$, respectively. The site indices $l$ and $m$, and the orbital index $\nu$ are defined as shown in Fig. 1 . In the following, we take both $a$ and $b$ as the unit of length and put $a=b=1$. $\mu$ represents the chemical potential. The noninteracting parts in eq. (1), i.e., $H_{\text {Ladder }}^{0}, H_{\text {Chain }}^{0}$, and $H_{\text {Ladder-Chain }}^{0}$, are represented by

$$
H_{\text {Ladder }}^{0}=
$$$$
\sum_{\boldsymbol{k} \sigma}\left(d_{1 \boldsymbol{k} \sigma}^{\dagger} d_{2 \boldsymbol{k} \sigma}^{\dagger} p_{1 \boldsymbol{k} \sigma}^{z \dagger} p_{1 \boldsymbol{k} \sigma}^{x \dagger} p_{2 \boldsymbol{k} \sigma}^{z \dagger} p_{2 \boldsymbol{k} \sigma}^{x \dagger} p_{3 \boldsymbol{k} \sigma}^{x \dagger}\right)\left(\begin{array}{ccccccc}
\varepsilon^{d p} & 0 & \zeta_{\boldsymbol{k}}^{z *} & 0 & 0 & \zeta_{\boldsymbol{k}}^{x *} & -\zeta_{\boldsymbol{k}}^{x} \\
0 & \varepsilon^{d p} & 0 & -\zeta_{\boldsymbol{k}}^{x} & \zeta_{\boldsymbol{k}}^{z *} & 0 & \zeta_{\boldsymbol{k}}^{x *} \\
\zeta_{\boldsymbol{k}}^{z} & 0 & 0 & 0 & 0 & \zeta_{\boldsymbol{k}}^{p *} & \zeta_{\boldsymbol{k}}^{p} \\
0 & -\zeta_{\boldsymbol{k}}^{x *} & 0 & 0 & \zeta_{\boldsymbol{k}}^{p *} & 0 & 0 \\
0 & \zeta_{\boldsymbol{k}}^{z} & 0 & \zeta_{\boldsymbol{k}}^{p} & 0 & 0 & \zeta_{\boldsymbol{k}}^{p *} \\
\zeta_{\boldsymbol{k}}^{x} & 0 & \zeta_{\boldsymbol{k}}^{p} & 0 & 0 & 0 & 0 \\
-\zeta_{\boldsymbol{k}}^{x *} & \zeta_{\boldsymbol{k}}^{x} & \zeta_{\boldsymbol{k}}^{p *} & 0 & \zeta_{\boldsymbol{k}}^{p} & 0 & 0
\end{array}\right)\left(\begin{array}{c}
d_{1 \boldsymbol{k} \sigma} \\
d_{2 \boldsymbol{k} \sigma} \\
p_{1 \boldsymbol{k} \sigma}^{z} \\
p_{1 \boldsymbol{k} \sigma}^{x} \\
p_{2 \boldsymbol{k} \sigma}^{z} \\
p_{2 \boldsymbol{k} \sigma}^{x} \\
p_{3 \boldsymbol{k} \sigma}^{x}
\end{array}\right),
$$$$
H_{\text {Chain }}^{0}=\sum_{\boldsymbol{k} \sigma}\left(d_{3 \boldsymbol{k} \sigma}^{\dagger} p_{4 \boldsymbol{k} \sigma}^{w \dagger} p_{4 \boldsymbol{k} \sigma}^{v \dagger} p_{5 \boldsymbol{k} \sigma}^{w \dagger} p_{5 \boldsymbol{k} \sigma}^{v \dagger}\right)\left(\begin{array}{ccccc}
\varepsilon^{d p}-\Delta V & -\xi_{\boldsymbol{k}}^{w} & \xi_{\boldsymbol{k}}^{v *} & \xi_{\boldsymbol{k}}^{w *} & -\xi_{\boldsymbol{k}}^{v} \\
-\xi_{\boldsymbol{k}}^{w *} & -\Delta V & \xi_{\boldsymbol{k}}^{z^{*}} & 0 & \xi_{\boldsymbol{k}}^{x *} \\
\xi_{\boldsymbol{k}}^{v} & \xi_{\boldsymbol{k}}^{z} & -\Delta V & \xi_{\boldsymbol{k}}^{x *} & 0 \\
\xi_{\boldsymbol{k}}^{w} & 0 & \xi_{\boldsymbol{k}}^{x} & -\Delta V & \xi_{\boldsymbol{k}}^{z} \\
-\xi_{\boldsymbol{k}}^{v *} & \xi_{\boldsymbol{k}}^{x} & 0 & \xi_{\boldsymbol{k}}^{z *} & -\Delta V
\end{array}\right)\left(\begin{array}{c}
d_{3 \boldsymbol{k} \sigma} \\
p_{4 \boldsymbol{k} \sigma}^{w} \\
p_{4 \boldsymbol{k} \sigma}^{v} \\
p_{5 \boldsymbol{k} \sigma}^{w} \\
p_{5 \boldsymbol{k} \sigma}^{v}
\end{array}\right)
$$

and

$$
H_{\text {Ladder-Chain }}^{0}=\sum_{\boldsymbol{k} \sigma}\left[\eta_{\boldsymbol{k}}^{+}\left(p_{4 \boldsymbol{k} \sigma}^{v \dagger} d_{2 \boldsymbol{k} \sigma}-p_{4 \boldsymbol{k} \sigma}^{w \dagger} d_{2 \boldsymbol{k} \sigma}\right)+\eta_{\boldsymbol{k}}^{-}\left(p_{5 \boldsymbol{k} \sigma}^{w \dagger} d_{1 \boldsymbol{k} \sigma}-p_{5 \boldsymbol{k} \sigma}^{v \dagger} d_{1 \boldsymbol{k} \sigma}\right)+\text { H.c. }\right],
$$

respectively. In eqs. (2)-(4), we use the abbreviations $\zeta_{\boldsymbol{k}}^{z}=2 \mathrm{i} t_{d p} \sin \frac{k_{z}}{2}, \zeta_{\boldsymbol{k}}^{x}=t_{d p} e^{-\mathrm{i} k_{x} / 2}, \zeta_{\boldsymbol{k}}^{p}=$ $2 \mathrm{i} t_{p p} e^{-\mathrm{i} k_{x} / 2} \sin \frac{k_{z}}{2}, \xi_{\boldsymbol{k}}^{w}=t_{d p} e^{\mathrm{i} k_{z} / 2} e^{\mathrm{i} k_{x} / 4}, \xi_{\boldsymbol{k}}^{v}=t_{d p} e^{\mathrm{i} k_{z} / 2} e^{-\mathrm{i} k_{x} / 4}, \xi_{\boldsymbol{k}}^{z}=t_{p p} e^{\mathrm{i} k_{z}}, \xi_{\boldsymbol{k}}^{x}=-t_{p p} e^{\mathrm{i} k_{x} / 2}-$ 
$2 t_{p p}^{\prime} e^{-\mathrm{i} k_{x}} \cos \frac{k_{z}}{2}$, and $\eta_{\boldsymbol{k}}^{ \pm}=2 \mathrm{i} t_{\perp} \sin \left(\frac{k_{y}}{2} \pm \frac{k_{z}}{4}\right)$. The transfer energies, $t_{d p}, t_{p p}, t_{p p}^{\prime}$, and $t_{\perp}$ are defined as shown in Fig. 1. The increase in $t_{\perp}$ in our model is considered to represent the increase in pressure in real Sr14-24-41. $\varepsilon^{d p}$ is the level difference between d- and p-electrons. Moreover, we use $\Delta V$ to control the charge imbalance between the ladder and chain layers. The change in $\Delta V$ can represent the change in Madelung energy due to the Ca doping of Sr1424-41. Considering only the on-site Coulomb repulsion among d-electrons, the interacting part $H^{\prime}$ in eq. (1) is described as

$$
H^{\prime}=\frac{U}{N} \sum_{l=1}^{3} \sum_{\boldsymbol{k} \boldsymbol{k}^{\prime} \boldsymbol{q}} d_{l \boldsymbol{k}+\boldsymbol{q} \uparrow}^{\dagger} d_{l \boldsymbol{k}^{\prime}-\boldsymbol{q} \downarrow}^{\dagger} d_{l \boldsymbol{k}^{\prime} \downarrow} d_{l \boldsymbol{k} \uparrow} .
$$

In eq. (5), $N$ is the number of $\boldsymbol{k}$-space lattice points in the first Brillouin zone (FBZ).

In the following analysis, we assume that only electrons on the Fermi surface of the same band can have pair instability. For our 3D d-p model, 2 or $3 d$-like bands intersect with the Fermi level. Thus, according to the Bardeen-Cooper-Schrieffer (BCS) theory, we have the following self-consistent equation for the pair function on the $\lambda$-th $d$-like band, $\Phi_{\boldsymbol{k}}^{\lambda}$ :

$$
\Phi_{\boldsymbol{k}}^{\lambda}=-\frac{1}{2 N} \sum_{i j \nu \boldsymbol{k}^{\prime}} V_{i j}\left(\boldsymbol{k}+\boldsymbol{k}^{\prime}\right) \frac{z_{i}^{\lambda}(\boldsymbol{k}) z_{j}^{\nu}\left(\boldsymbol{k}^{\prime}\right)}{\sqrt{\left(\varepsilon_{\boldsymbol{k}^{\prime}}^{\nu}-\mu\right)^{2}+\left(\Phi_{\boldsymbol{k}^{\prime}}^{\nu}\right)^{2}}} \Phi_{\boldsymbol{k}^{\prime}}^{\nu},
$$

where $i, j=1,2,3$ (Cu site indices) and $\lambda, \nu=1,2(, 3)$ ( $d$-like band indices). This equation is valid for both the spin-singlet and spin-triplet pair functions if we define $V_{i j}(\boldsymbol{q})$ differently as the need arises. Thus, hereafter, we omit the spin indices. $V_{i j}(\boldsymbol{q})$ represents the effective pair scattering between a d-electron on the $i$-th site and one on the $j$-th site. The term $\varepsilon_{\boldsymbol{k}}^{\nu}$ represents the energy dispersion of the $\nu$-th $d$-like band, and $z_{i}^{\lambda}(\boldsymbol{k})$ represents the matrix element of unitary transformation. These variables are obtained by solving the eigen-equation for the noninteracting part $H_{\text {Ladder }}^{0}+H_{\text {Chain }}^{0}+H_{\text {Ladder-Chain }}^{0}$ in eq. (1). We set $\Phi_{\boldsymbol{k}}^{\lambda}=\Delta_{\mathrm{sc}} \cdot \Psi_{\boldsymbol{k}}^{\lambda}$, where $\Delta_{\text {sc }}$ denotes the magnitude of $\Phi_{k}^{\lambda}$, and $\Psi_{k}^{\lambda}$ represents its $k$ dependence on the $\lambda$-th $d$-like band. On the basis of Kondo's argument, ${ }^{35)}$ retaining only the divergent term, we can rewrite eq. (6) as

$$
\Psi_{\boldsymbol{k}}^{\lambda}=\log _{e} \Delta_{\mathrm{sc}} \cdot \frac{1}{N} \sum_{i j \nu \boldsymbol{k}^{\prime}} V_{i j}\left(\boldsymbol{k}+\boldsymbol{k}^{\prime}\right) z_{i}^{\lambda}(\boldsymbol{k}) z_{j}^{\nu}\left(\boldsymbol{k}^{\prime}\right) \delta\left(\varepsilon_{\boldsymbol{k}^{\prime}}^{\nu}-\mu\right) \Psi_{\boldsymbol{k}^{\prime}}^{\nu}
$$

for a very small $\Delta_{\mathrm{sc}}$. Equation (7) is a homogeneous integral equation for $\Psi_{\boldsymbol{k}}^{\lambda}$ with an eigenvalue of $1 / \log \Delta_{\mathrm{sc}}$. We are interested in obtaining the most stable pairing state, so we must find the eigenvector $\Psi_{k}^{\lambda}$ with the smallest eigenvalue $1 / \log \Delta_{\mathrm{sc}}$ using eq. (7) when $\Delta_{\mathrm{sc}}$ is maximum. Given the quasi-two-dimensionality of our 3D d-p model, i.e., $t_{\perp} \ll t_{d p}$, we assume five functions as candidates for the most stable pairing state:

$$
a^{\lambda}: s \text {-wave-like singlet, }
$$


J. Phys. Soc. Jpn.

Full PaPer

$\sum_{m_{z}=1}^{M} a_{z}^{\lambda}\left(m_{z}\right) \cos m_{z} k_{z}+\sum_{m_{z}=1}^{M} \sum_{m_{x}=1}^{2 m_{z}} a_{x}^{\lambda}\left(m_{z} m_{x}\right) \cos \frac{2 m_{z}-m_{x}}{2} k_{z} \cos \frac{3 m_{x}}{2} k_{x}+\sum_{m_{y}=1}^{M} a_{y}^{\lambda}\left(m_{y}\right) \cos m_{y} k_{y}$

$: d_{z^{2}-x^{2}}$-wave-like singlet,

$\sum_{m_{z}=1}^{M} \sum_{m_{x}=1}^{2 m_{z}-1} a^{\lambda}\left(m_{z} m_{x}\right) \sin \frac{2 m_{z}-m_{x}}{2} k_{z} \sin \frac{3 m_{x}}{2} k_{x}: d_{z x}$-wave-like singlet,

$\sum_{m_{z}=1}^{M} a^{\lambda}\left(m_{z}\right) \sin m_{z} k_{z}: p_{z}$-wave-like triplet,

$\sum_{m_{z}=1}^{M} \sum_{m_{x}=1}^{2 m_{z}} a^{\lambda}\left(m_{z} m_{x}\right) \cos \frac{2 m_{z}-m_{x}}{2} k_{z} \sin \frac{3 m_{x}}{2} k_{x}: p_{x}$-wave-like triplet.

In order to solve eq. (7), we substitute these candidates for $\Psi_{\boldsymbol{k}}^{\lambda}$ and $\Psi_{\boldsymbol{k}^{\prime}}^{\nu}$, and integrate for $k_{z}$, $k_{z}^{\prime}, k_{x}, k_{x}^{\prime}, k_{y}$, and $k_{y}^{\prime}$. Then, we can safely reduce our original eigenvalue problem for $\Psi_{\boldsymbol{k}}^{\lambda}$ to an eigenvalue problem for $a^{\lambda}\left[a_{z}^{\lambda}\left(m_{z}\right), a_{x}^{\lambda}\left(m_{z} m_{x}\right)\right.$, and $\left.a_{y}^{\lambda}\left(m_{y}\right)\right]$ in order to obtain the most stable pairing state. When we solve it numerically by the standard method, we obtain both the eigenvalue $1 / \log _{e} \Delta_{\mathrm{sc}}$ and the eigenvector $a^{\lambda}\left[a_{z}^{\lambda}\left(m_{z}\right), a_{x}^{\lambda}\left(m_{z} m_{x}\right)\right.$, and $\left.a_{y}^{\lambda}\left(m_{y}\right)\right]$. Here, to solve eq. (7) within SOPT for singlet-pairing states, we have

$$
V_{i j}(\boldsymbol{q})=U \delta_{i j}+\frac{U^{2}}{N} \sum_{\kappa \rho \boldsymbol{k}} z_{i}^{\kappa}(\boldsymbol{q}+\boldsymbol{k}) z_{j}^{\rho}(\boldsymbol{k}) \frac{\left(1-f_{\boldsymbol{q}+\boldsymbol{k}}^{\kappa}\right) f_{\boldsymbol{k}}^{\rho}}{\varepsilon_{\boldsymbol{q}+\boldsymbol{k}}^{\kappa}-\varepsilon_{\boldsymbol{k}}^{\rho}} .
$$

Meanwhile, for triplet-pairing states,

$$
V_{i j}(\boldsymbol{q})=-\frac{U^{2}}{N} \sum_{\kappa \rho \boldsymbol{k}} z_{i}^{\kappa}(\boldsymbol{q}+\boldsymbol{k}) z_{j}^{\rho}(\boldsymbol{k}) \frac{\left(1-f_{\boldsymbol{q}+\boldsymbol{k}}^{\kappa}\right) f_{\boldsymbol{k}}^{\rho}}{\varepsilon_{\boldsymbol{q}+\boldsymbol{k}}^{\kappa}-\varepsilon_{\boldsymbol{k}}^{\rho}},
$$

where

$$
f_{\boldsymbol{k}}^{\rho}=\frac{1}{2}\left[1-\tanh \left(\frac{\varepsilon_{\boldsymbol{k}}^{\rho}-\mu}{2 T}\right)\right],
$$

and $T$ denotes the temperature.

\section{Results and Discussion}

In our present analyses, all $\varepsilon_{\boldsymbol{k}}^{\nu}$ and $z_{i}^{\lambda}(\boldsymbol{k})$ in eq. (7) are first calculated for the $\boldsymbol{k}$-points on an equally spaced mesh in FBZ for each band. The mesh size along $k_{z}$ is 108 , and the sizes along $k_{x}$ and $k_{y}$ are both 64 . Then, we calculate $V_{i j}\left(\boldsymbol{k}+\boldsymbol{k}^{\prime}\right)$ in eq. (7) only for $\boldsymbol{k}$ - and $\boldsymbol{k}^{\prime}$-points satisfying the conditions $\varepsilon_{\boldsymbol{k}}^{\lambda}=\mu$ and $\varepsilon_{\boldsymbol{k}^{\prime}}^{\nu}=\mu$, respectively. When we calculate $V_{i j}\left(\boldsymbol{k}+\boldsymbol{k}^{\prime}\right)$ according to eqs. (12) and (14), we set the temperature $T=0.001 \mathrm{eV} \approx 10 \mathrm{~K}$, at which our system can be considered to behave similarly to the ground state. These calculations have been performed at $U=0.3 \mathrm{eV}$, where magnetic instabilities cannot occur. Other common parameters are $t_{d p}=1.00 \mathrm{eV}, t_{p p}=-0.50 \mathrm{eV}, t_{p p}^{\prime}=-0.10 \mathrm{eV}$, and $\varepsilon^{d p}=2.60 \mathrm{eV}$. These parameters are determined using examples from studies of the three-band Hubbard ladder 
model,${ }^{10,20}$ local-density approximation, ${ }^{37)}$ and angle-resolved photo-emission spectroscopy (ARPES). ${ }^{38-40)}$

As mentioned in the last section, varying the pressure and Ca doping in real Sr14-24-41 can be reproduced by changing $t_{\perp}$ and $\Delta V$, respectively, in our model. Thus, in order to comprehensively understand the superconductivity of spin-chain ladder cuprate, we calculate the $\log _{e} \Delta_{\mathrm{sc}}$ of the most stable superconducting state for various $t_{\perp}$ and $\Delta V$ values. All through these calculations, we fix average holes for $\mathrm{Cu}^{2+}\left[n^{\mathrm{h}}\left(\mathrm{Cu}^{2+}\right)\right]$ to 0.25 as well as the value of real Sr14-24-41. ${ }^{1)}$ Here, $n^{\mathrm{h}}\left(\mathrm{Cu}^{2+}\right)$ is defined as $n^{\mathrm{h}}\left(\mathrm{Cu}^{2+}\right) \equiv\left[2 n_{\text {Ladder }}^{\mathrm{h}}\left(\mathrm{Cu}^{2+}\right)+n_{\text {Chain }}^{\mathrm{h}}\left(\mathrm{Cu}^{2+}\right)\right] / 3$, where $n_{\text {Ladder }}^{\mathrm{h}}\left(\mathrm{Cu}^{2+}\right)$ and $n_{\text {Chain }}^{\mathrm{h}}\left(\mathrm{Cu}^{2+}\right)$ are average holes per $\mathrm{Cu}^{2+}$ in the ladder and chain layers, respectively. These values should be estimated as the total holes in our model in order to be compared with those of real Sr14-24-41.1) We find that the most stable superconducting state of our five candidates is always the $p_{z}$-wave-like triplet, represented by eq. (10). We confirm that $\log _{e} \Delta_{\text {sc }}$ estimated for this state with $M=16$ does not differ from that with $M=15$ by more than $8 \%$. The following discussion is therefore restricted to the $p_{z}$-wave-like triplet state with $M=16$.

In Fig. 2, we summarize how $\log _{e} \Delta_{\text {sc }}, n_{\text {Ladder }}^{\mathrm{h}}\left(\mathrm{Cu}^{2+}\right)$ and $n_{\text {Chain }}^{\mathrm{h}}\left(\mathrm{Cu}^{2+}\right)$ depend on $t_{\perp}$ for various $\Delta V$ values. Figure 2(a) shows the $\log _{e} \Delta_{\mathrm{sc}}$ dependence on $t_{\perp}$ for $\Delta V=-0.12 \mathrm{eV}$ and $\Delta V=-0.11 \mathrm{eV}$, as well as the $T_{\mathrm{c}}$ dependence on pressure in real Sr14-24-41, ${ }^{25-28)}$ i.e., it has a maximum at an intermediate $t_{\perp}$. Since the temperature $T=0.001 \mathrm{eV} \approx 10 \mathrm{~K}$ is thought to be sufficiently low, $T_{\mathrm{c}}$ should be proportional to $\Delta_{\mathrm{sc}}(T)$ within the BCS theory. Thus, our results of the $\log _{e} \Delta_{\mathrm{sc}}$ dependence on $t_{\perp}$ for $\Delta V=-0.12 \mathrm{eV}$ and $\Delta V=-0.11 \mathrm{eV}$ can qualitatively reproduce the $T_{\mathrm{c}}$ dependence on pressure in real Sr14-24-41. Moreover, as shown in Figs. 2(b) and $2(\mathrm{c}), \mathrm{Cu}^{2+}$ holes are transferred from the chain layer to the ladder layer with an increase in $t_{\perp}$, which also qualitatively agrees with the experimental results under high pressures. ${ }^{25,34)}$

On the other hand, for $\Delta V=-0.10 \mathrm{eV}, \log _{e} \Delta_{\mathrm{sc}}$ increases with an increase in $t_{\perp}$. For $\Delta V=-0.09 \mathrm{eV}, \log _{e} \Delta_{\mathrm{sc}}$ decreases with an increase in $t_{\perp}$, but it tends to be much larger than those in the other cases. The fact that the behavior of $\log _{e} \Delta_{\mathrm{sc}}$ differs depending on $\Delta V$ can be explained by the configuration of the Fermi surface and the density of state (DOS) on it. Let us show the DOS's on the Fermi surface for $\Delta V=-0.12,-0.11,-0.10$, and $-0.09 \mathrm{eV}$ in Figs. 3-6, respectively. For $\Delta V=-0.12,-0.11$, and $-0.10 \mathrm{eV}$, we have two quasi-one-dimensional (Q1D) branches of the Fermi surface, which we call "Branch 1" and "Branch 2", as shown in Figs. 3-5, respectively. Branch 2 becomes increasingly warped as $t_{\perp}$ increases, and simultaneously the DOS on the branch is enhanced. A large DOS is favorable for superconductivity. However, the warped Fermi surface makes Fermi surface nesting worse. The effective pair scattering $V_{i j}(\boldsymbol{q})$, defined by eqs. (12) and (14), is mainly enhanced by Fermi surface nesting; thus, the warped Fermi surface suppresses pair instability. Owing to these two conflicting effects on the superconductivity, the $\log _{e} \Delta_{\mathrm{sc}}$ values for $\Delta V=-0.12$ 

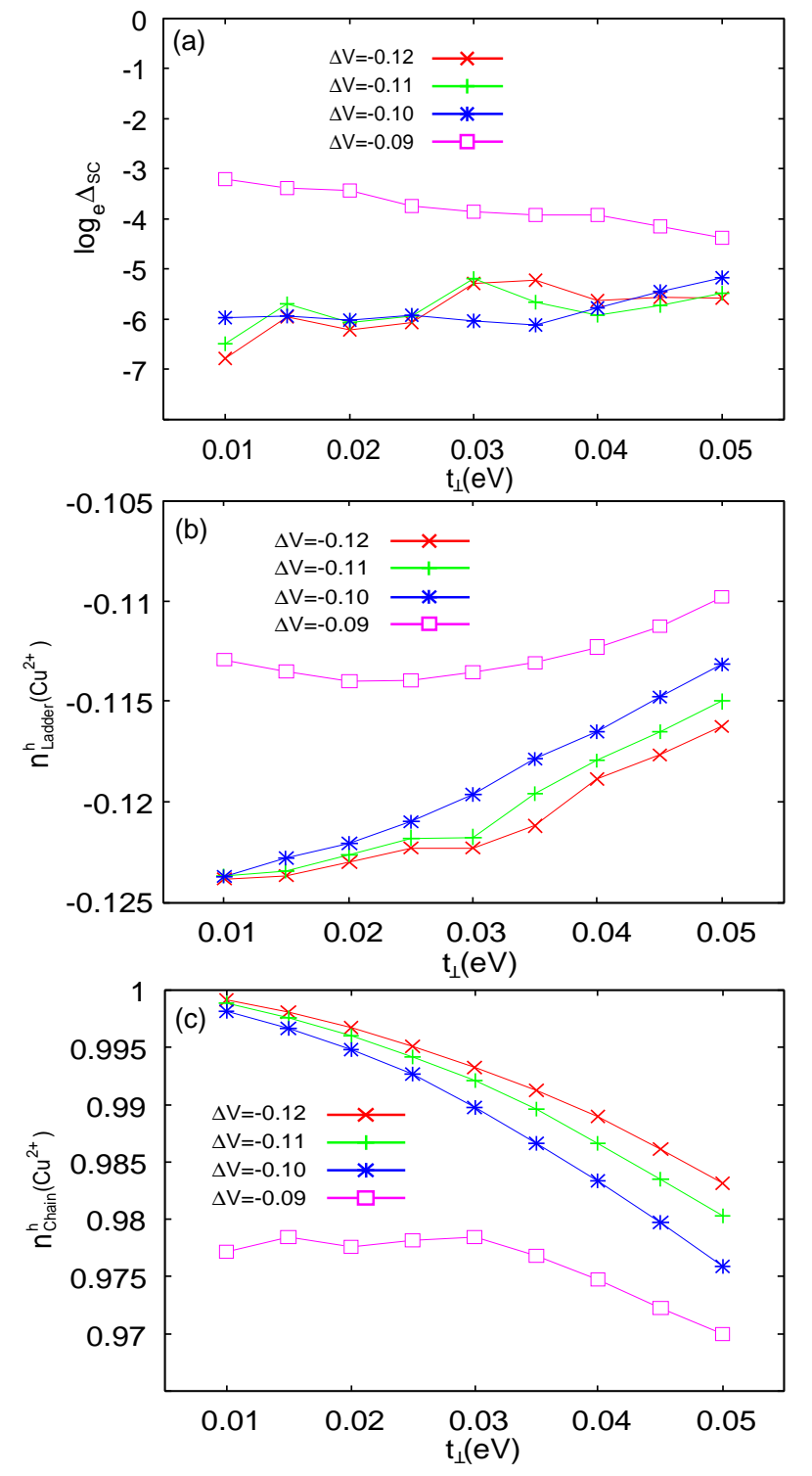

Fig. 2. (Color online) (a) $\log \Delta_{\mathrm{sc}}$, (b) $n_{\text {Ladder }}^{\mathrm{h}}\left(\mathrm{Cu}^{2+}\right)$, and (c) $n_{\text {Chain }}^{\mathrm{h}}\left(\mathrm{Cu}^{2+}\right)$ for $\Delta V=-0.12,-0.11$, -0.10 , and $-0.09 \mathrm{eV}$.

and $-0.11 \mathrm{eV}$ have maxima at approximately $t_{\perp} \approx 0.035$ and $0.030 \mathrm{eV}$, respectively, when $t_{\perp}$ varies. Although this situation on the Fermi surface is common for $\Delta V=-0.10 \mathrm{eV}$, the DOS on Branch 2 is enhanced more rapidly with $t_{\perp}$, as shown in Fig. 5 (note that the color bar scale in Fig. 5 is about twice as large as those in Figs. 3 and 4), and such a large DOS surpasses the other effect due to the warped Fermi surface in this case. This is the reason why $\log _{e} \Delta_{\mathrm{sc}}$ for $\Delta V=-0.10 \mathrm{eV}$ increases with an increase in $t_{\perp}$. On the other hand, for $\Delta V=-0.09 \mathrm{eV}$, we have three branches of the Fermi surface. Of these, two are Q1D branches and are called similarly to those for $\Delta V=-0.12 \mathrm{eV}$. The other branch of the Fermi surface is a quasi-twodimensional (Q2D) branch, newly labeled "Branch 3" in Figs. 6(a) and 6(b). Noting that the 


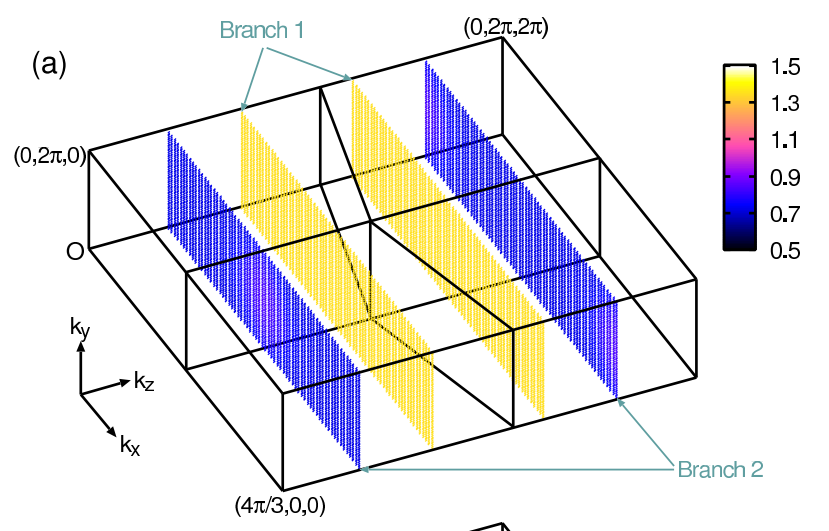

(b)

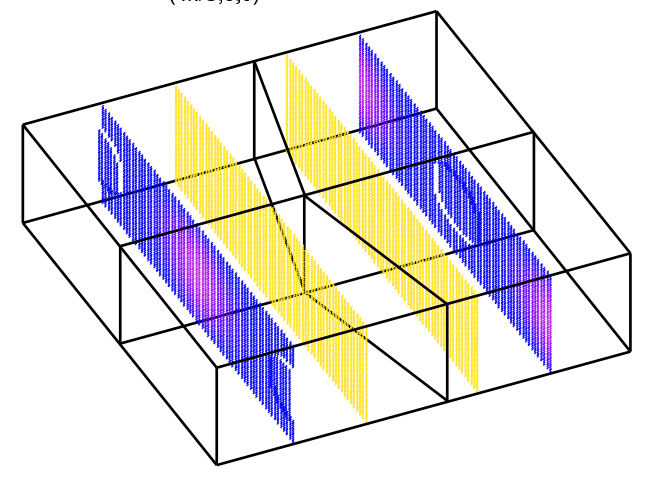

(c)

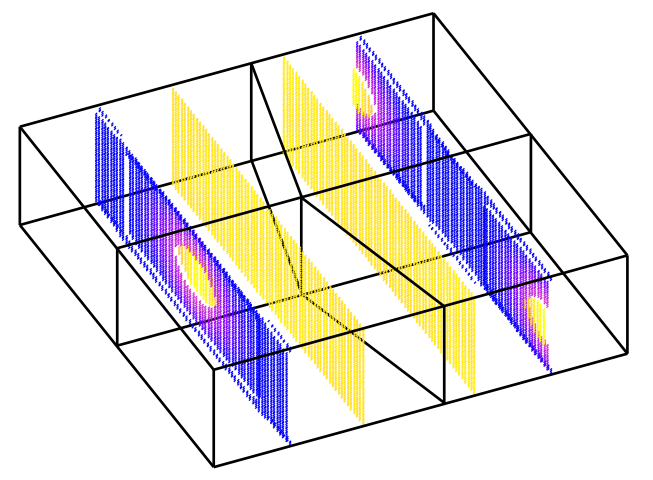

Fig. 3. (Color online) Densities of states (DOS's) on Fermi surface for $\Delta V=-0.12 \mathrm{eV}$ : (a) for $t_{\perp}=0.010 \mathrm{eV}$, (b) for $t_{\perp}=0.030 \mathrm{eV}$, and (c) for $t_{\perp}=0.050 \mathrm{eV}$.

color bar scale in Fig. 6 is about 100/3 times as large as that in Fig. 5, Branch 3 has an extremely large DOS compared with the other branches regardless of $t_{\perp}$. This is why $\log _{e} \Delta_{\mathrm{sc}}$ for $\Delta V=-0.09 \mathrm{eV}$ always becomes large. However, as shown in Figs. 6(b) and 6(c), Branch 3 becomes smaller and finally disappears as $t_{\perp}$ increases. This leads to a decrease in total DOS and the degradation of superconductivity.

When we compare the Fermi surface obtained by our present calculation with that observed by ARPES, ${ }^{40)}$ we find that the results for $\Delta V=-0.12,-0.11$, and $-0.10 \mathrm{eV}$ are similar. In other words, in real Sr14-24-41, the charge imbalance between the ladder and chain is too large to have three branches of the Fermi surface, as observed in our results for $\Delta V=-0.09 \mathrm{eV}$. Thus, the superconductivity in spin-chain ladder cuprate may be enhanced 


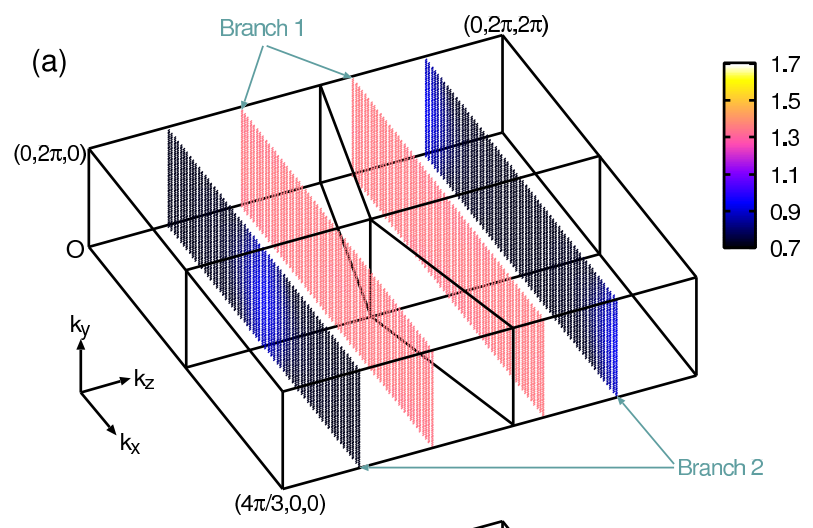

(b)

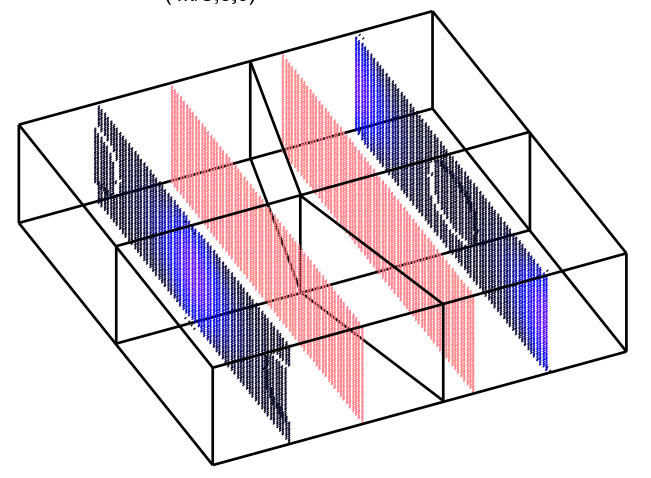

(c)

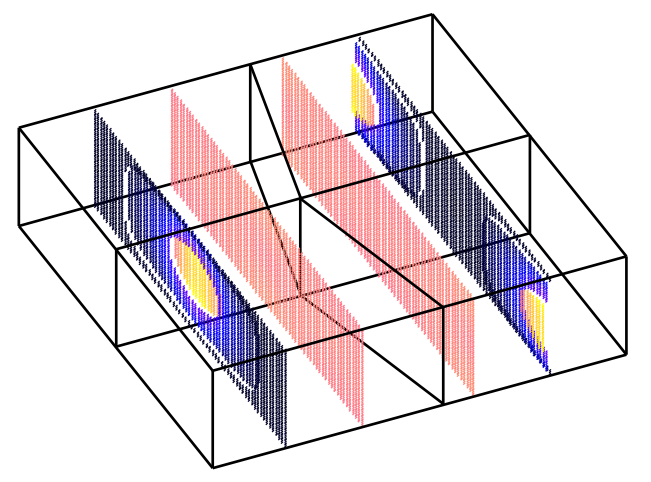

Fig. 4. (Color online) DOS's on Fermi surface for $\Delta V=-0.11 \mathrm{eV}$ : (a) for $t_{\perp}=0.010 \mathrm{eV}$, (b) for $t_{\perp}=0.030 \mathrm{eV}$, and (c) for $t_{\perp}=0.050 \mathrm{eV}$.

if the large charge imbalance between the ladder and the chain can be resolved, for example, by varying the elements of the spacer layer without changing their valences.

Hereafter, we discuss the gap functions of the $p_{z}$-wave-like superconducting state in detail. $\Psi_{\boldsymbol{k}}^{\lambda}$ on the Fermi surface for $\Delta V=-0.12 \mathrm{eV}$ and for $\Delta V=-0.09 \mathrm{eV}$ are shown in Figs. 7 and 8 , respectively. For $\Delta V=-0.12 \mathrm{eV}, \Psi_{\boldsymbol{k}}^{\lambda}$ changes its sign on the disconnected parts of Branch 1, and it does so on those of Branch 2, as shown in Fig. 7. This is similar to the triplet state derived by Sasaki et al. ${ }^{32)}$ Furthermore, $\Psi_{\boldsymbol{k}}^{\lambda}$ has no nodes on the connected parts of each branch. Thus, this superconducting state is expected to behave as a fully gapped state for the microscopically experimental probe. Actually, the relaxation rate $T_{1}^{-1}$ of the NMR measurement suggests that the superconducting state of Sr14-24-41 at pressures of 3.5-3.8GPa 


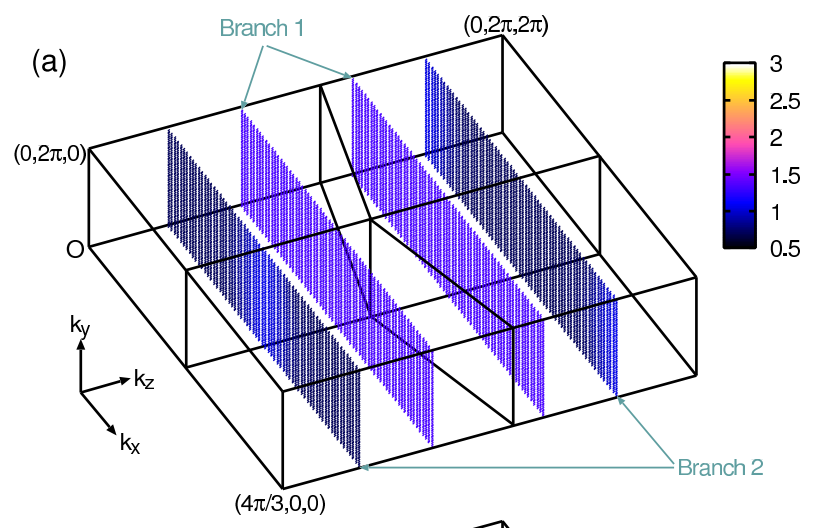

(b)

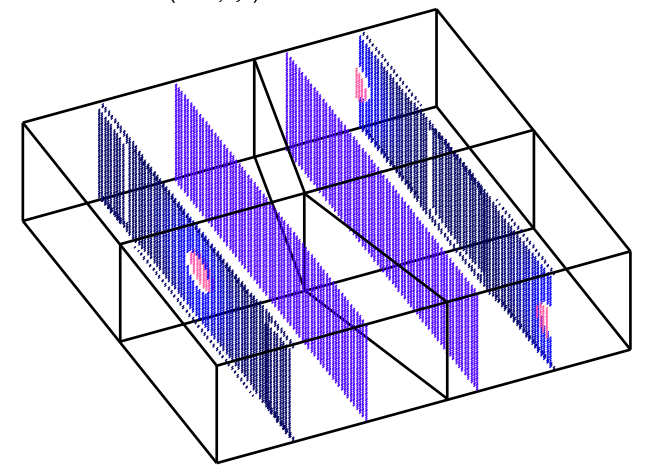

(c)

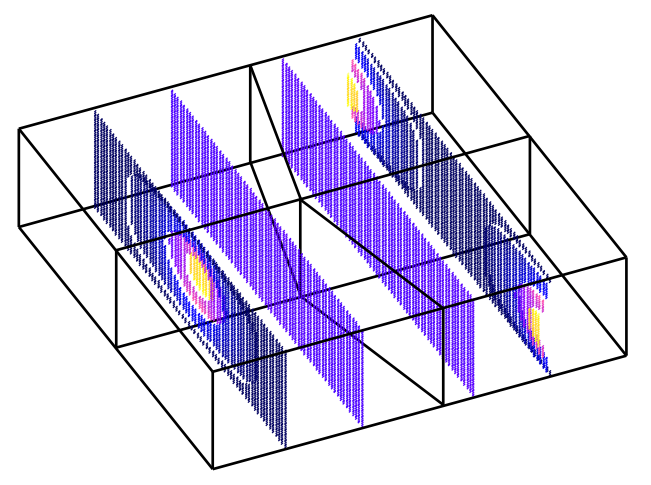

Fig. 5. (Color online) DOS's on Fermi surface for $\Delta V=-0.10 \mathrm{eV}$ : (a) for $t_{\perp}=0.010 \mathrm{eV}$, (b) for $t_{\perp}=0.030 \mathrm{eV}$, and $(\mathrm{c})$ for $t_{\perp}=0.050 \mathrm{eV}$.

has an $s$-wave-like character. ${ }^{41,42)}$ Moreover, in their works, the Knight shift of ${ }^{63} \mathrm{Cu}$ nuclei for the ladder derived from high fields shows no change below $T_{\mathrm{c}}$. This fact strongly suggests that the superconducting state in real Sr14-24-41 can be a singlet-Fulde-Ferrell-Larkin-Ovchinnikov (FFLO) state or a triplet superconducting state, and the latter is consistent with our result for $\Delta V=-0.12 \mathrm{eV}$.

However, the fully gapped superconductivity for $\Delta V=-0.12 \mathrm{eV}$ does not remain for $\Delta V=-0.09 \mathrm{eV}$. As shown in Fig. $8, \Psi_{\boldsymbol{k}}^{\lambda}$ has nodes on Branch 3 or 2 . These results indicate that our $p_{z}$-wave-like gap function is not robust in terms of the absence of nodes. If the NMR measurement can proceed in Sr14-24-41 under different conditions, we may observe $T_{1}^{-1}$ for the gapless superconductivity. 

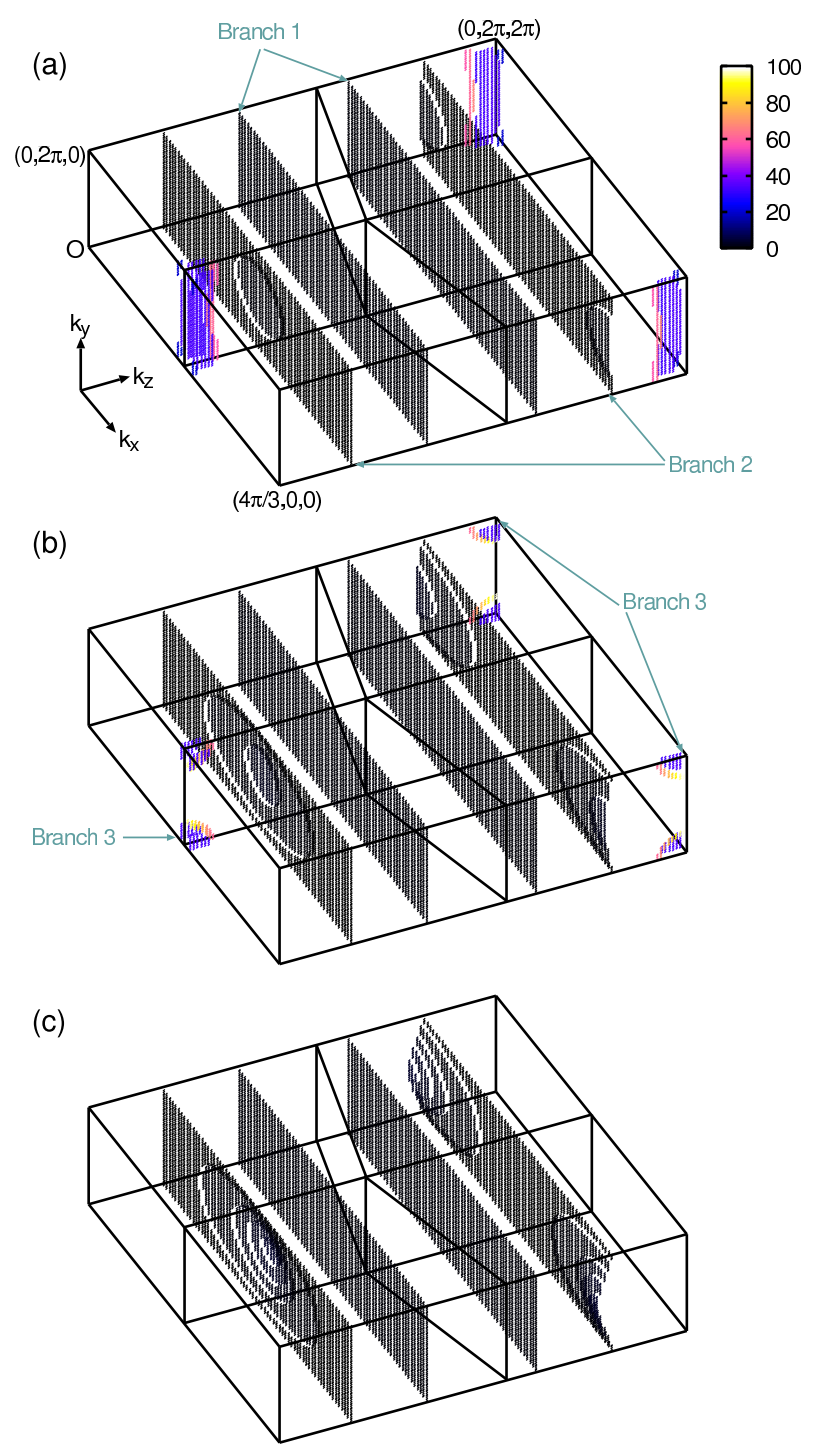

Fig. 6. (Color online) DOS's on Fermi surface for $\Delta V=-0.09 \mathrm{eV}$ : (a) for $t_{\perp}=0.010 \mathrm{eV}$, (b) for $t_{\perp}=0.030 \mathrm{eV}$, and (c) for $t_{\perp}=0.050 \mathrm{eV}$.

Finally, note that the amplitude of $\Psi_{k}^{\lambda}$ on Branch 3 is smaller than that on Branch 1 or 2 , as shown in Fig. 8. The large DOS on Branch 3 mainly enhances the superconductivity on the other branches, not on itself. This synergistic effect is caused by the interband interaction, i.e., $V_{i j}\left(\boldsymbol{k}+\boldsymbol{k}^{\prime}\right) z_{i}^{\lambda}(\boldsymbol{k}) z_{j}^{\nu}\left(\boldsymbol{k}^{\prime}\right)$ for $\lambda \neq \nu$ in eq. (6), originating from the mixing between dand p-orbitals. Thus, the hybridization effect also plays a significant role in enhancing the superconductivity in this material.

\section{Conclusions}

We have demonstrated that the 3D d-p model with the Q1D structure similar to Sr14-24-41 can represent $p_{z}$-wave-like triplet superconductivity up to the second order in the perturbation theory framework. On the basis of this model, we can reproduce the $T_{\mathrm{c}}$ dependence on pressure 


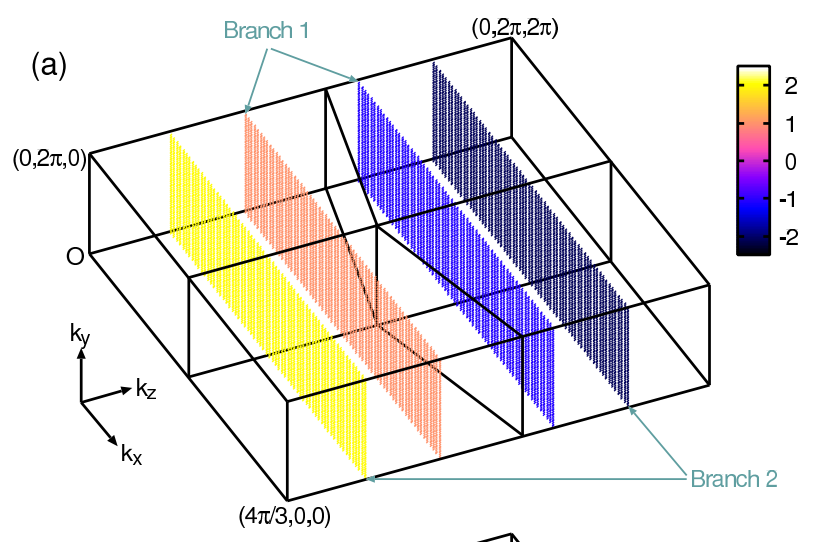

(b)

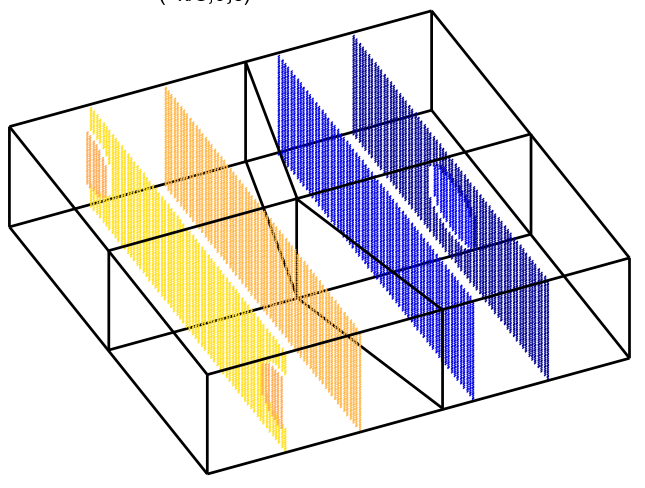

(c)

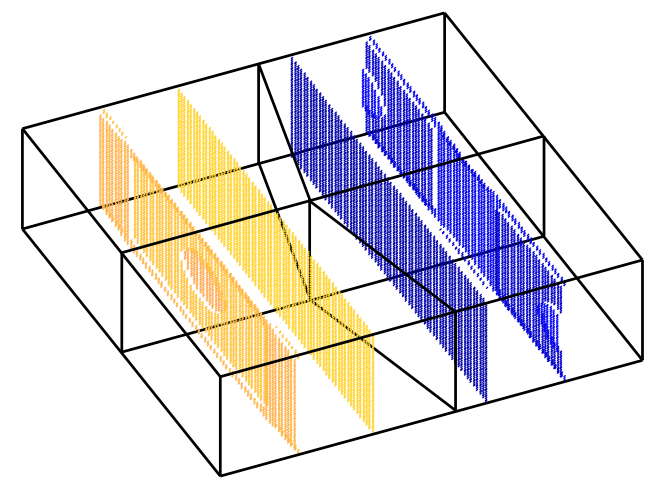

Fig. 7. (Color online) $\Psi_{\boldsymbol{k}}^{\lambda}$ on the Fermi surface for $\Delta V=-0.12 \mathrm{eV}$ : (a) for $t_{\perp}=0.010 \mathrm{eV}$, (b) for $t_{\perp}=0.030 \mathrm{eV}$, and $(\mathrm{c})$ for $t_{\perp}=0.050 \mathrm{eV}$.

by changing the interlayer coupling. The calculated results on the Fermi surface configuration and the superconducting state can give a comprehensive picture to explain the ARPES and NMR experimental results. Moreover, our results show the possibility of enhancing the superconductivity if the charge imbalance between the ladder and the chain can be decreased by varying the elements of the spacer layer. This speculation is based on the fact that the hybridization effect due to interlayer coupling and the other mixing between d- and p-orbitals is crucial to enhance the superconductivity. 
J. Phys. Soc. Jpn.

Full PAPER
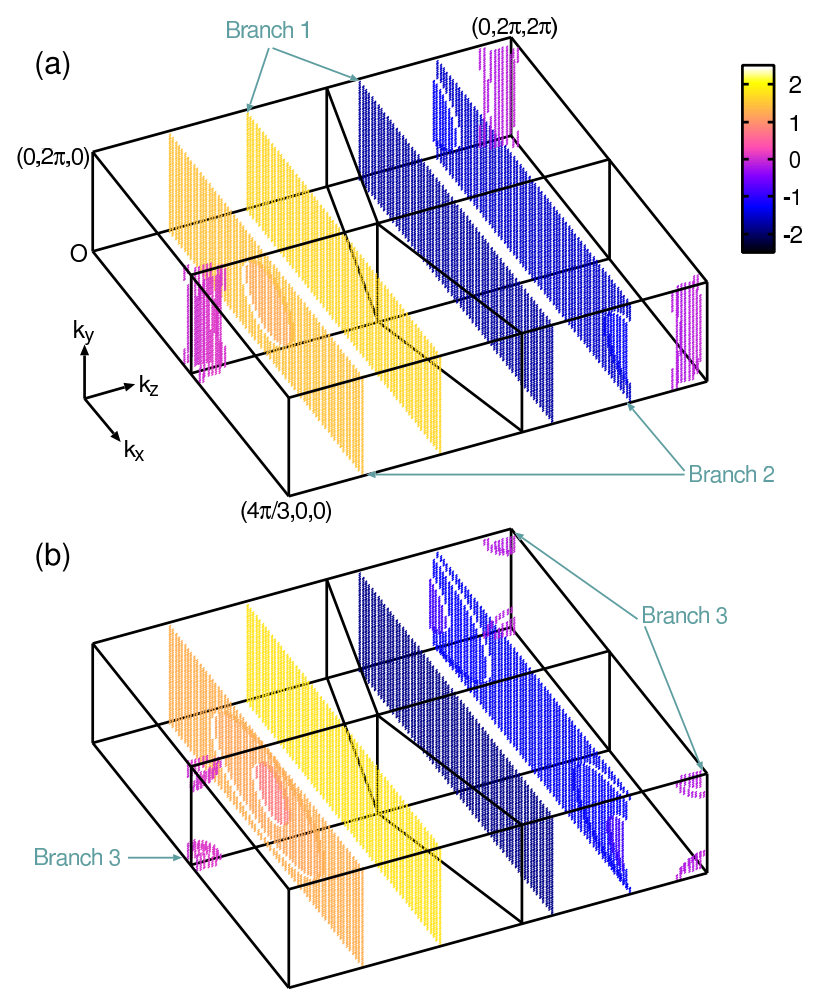

(c)

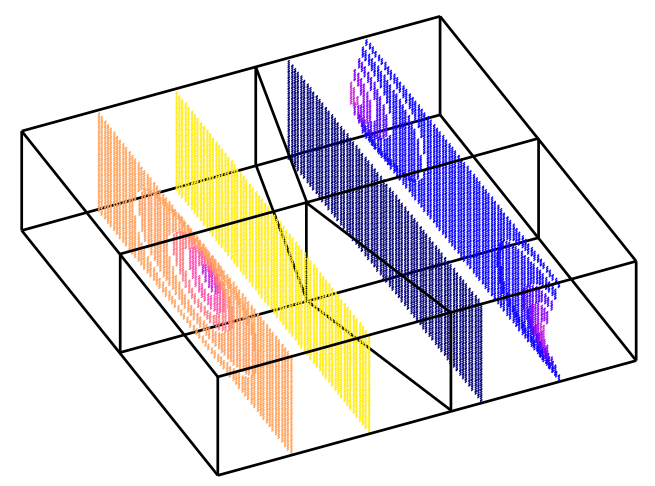

Fig. 8. (Color online) $\Psi_{k}^{\lambda}$ on the Fermi surface for $\Delta V=-0.09 \mathrm{eV}$ : (a) for $t_{\perp}=0.010 \mathrm{eV}$, (b) for $t_{\perp}=0.030 \mathrm{eV}$, and (c) for $t_{\perp}=0.050 \mathrm{eV}$.

\section{Acknowledgments}

The authors are grateful to Professors K. Yamaji and I. Hase for helpful discussions. The authors also thank the referee for invaluable comments. 
J. Phys. Soc. Jpn.

Full PAPER

\section{References}

1) E. M. McCarron, III, M. A. Subramanian, J. C. Calabrese, and R. L. Harlow: Mat. Res. Bull. 23 (1988) 1355.

2) T. Siegrist, L. F. Schneemeyer, S. A. Sunshine, J. V. Waszezak, and R. S. Roth: Mat. Res. Bull. 23 (1988) 1429.

3) M. Kato, H. Chizawa, Y. Koike, T. Noji, and Y. Saito: Physica C 235-240 (1994) 1327.

4) E. Dagotto and T. M. Rice: Science 271 (1996) 618.

5) E. Dagotto, J. Riera, and D. Scalapino: Phys. Rev. B 45 (1992) 5744.

6) T. M. Rice, S. Gopalan, and M. Sigrist: Europhys. Lett. 23 (1993) 445.

7) M. Sigrist, T. M. Rice, and F. C. Zhang: Phys. Rev. B 49 (1994) 12058.

8) H. Tsunetsugu, M. Troyer, and T. M. Rice: Phys. Rev. B 49 (1994) 16078.

9) C. A. Hayward, D. Poilblanc, R. M. Noack, D. J. Scalapino, and W. Hanke: Phys. Rev. Lett. 75 (1995) 926.

10) E. Jeckelmann, D. J. Scalapino, and S. R. White: Phys. Rev. B 58 (1998) 9492.

11) K. Yamaji and Y. Shimoi: Physica C 222 (1994) 349.

12) D. V. Khveshchenko and T. M. Rice: Phys. Rev. B 50 (1994) 252.

13) R. M. Noack, S. R. White, and D. J. Scalapino: Phys. Rev. Lett. 73 (1994) 882; Europhys. Lett. 30 (1995) 163.

14) Y. Asai: Phys. Rev. B 50 (1994) 6519.

15) T. Yanagisawa, Y. Shimoi, and K. Yamaji: Phys. Rev. B 52 (1995) R3860.

16) L. Balents and M. P. A. Fisher: Phys. Rev. B 53 (1996) 12133.

17) K. Kuroki, T. Kimura, and H. Aoki: Phys. Rev. B 54 (1996) R15641.

18) T. Dahm and D. J. Scalapino: Physica C 288 (1997) 33.

19) S. Koike, K. Yamaji, and T. Yanagisawa: J. Phys. Soc. Jpn. 68 (1999) 1657; 69 (2000) 2199.

20) S. Nishimoto, E. Jeckelmann and D. J. Scalapino: Phys. Rev. B 66 (2002) 245109; 79 (2009) 205115.

21) M. Uehara, T. Nagata, J. Akimitsu, H. Takahashi, N. Môri, and K. Kinoshita: J. Phys. Soc. Jpn. 65 (1996) 2764.

22) M. Kato, K. Shiota, and Y. Koike: Physica C 258 (1996) 284.

23) N. Motoyama, T. Osafune, T. Kakeshita, H. Eisaki, and S. Uchida: Phys. Rev. B 55 (1997) R3386.

24) T. Osafune, N. Motoyama, H. Eisaki, and S. Uchida: Phys. Rev. Lett. 78 (1997) 1980.

25) M. Isobe, T. Ohta, M. Onoda, F. Izumi, S. Nakano, J. Q. Li, Y. Matsui, E. Takayama-Muromachi, T. Matsumoto, and H. Hayakawa: Phys. Rev. B 57 (1998) 613.

26) T. Nagata, M. Uehara, J. Goto, J. Akimitsu, N. Motoyama, H. Eisaki, S. Uchida, H. Takahashi, T. Nakanishi, and N. Môri: Phys. Rev. Lett. 81 (1998) 1090.

27) H. Eisaki, N. Motoyama, K. M. Kojima, S. Uchida, T. Nagata, N. Takeshita, and N. Môri: Physica C 341-348 (2000) 363.

28) N. Motoyama, H. Eisaki, S. Uchida, N. Takeshita, N. Môri, T. Nakanishi, and H. Takahashi: Europhys. Lett. 58 (2002) 758.

29) K. M. Kojima, N. Motoyama, H. Eisaki, and S. Uchida: J. Electron Spectrosc. 117-118 (2001) 237.

30) J. Kishine and K. Yonemitsu: J. Phys. Soc. Jpn. 66 (1997) 3725. 
31) H. Kontani and K. Ueda: Phys. Rev. Lett. 80 (1998) 5619.

32) S. Sasaki, H. Ikeda, and K. Yamada: J. Phys. Soc. Jpn. 73 (2004) 2822.

33) K. Kuroki, T. Higashida, and R. Arita: Phys. Rev. B 72 (2005) 212509.

34) M. Isobe, M. Onoda, T. Ohta, F. Izumi, K. Kimoto, E. Takayama-Muromachi, A. W. Hewat, and K. Ohoyama: Phys. Rev. B 62 (2000) 11667.

35) J. Kondo: J. Phys. Soc. Jpn. 70 (2001) 808.

36) S. Koikegami and T. Yanagisawa: J. Phys. Soc. Jpn. 75 (2006) 034715.

37) M. Arai and H. Tsunetsugu: Phys. Rev. B 56 (1997) R4305.

38) T. Takahashi, T. Yokoya, A. Ashihara, O. Akaki, H. Fujisawa, A. Chainani, M. Uehara, T. Nagata, J. Akimitsu, and H. Tsunetsugu: Phys. Rev. B 56 (1997) 7870.

39) T. Sato, T. Yokoya, T. Takahashi, M. Uehara, T. Nagata, J. Goto, and J. Akimitsu: J. Phys. Chem Solids 59 (1998) 1912.

40) T. Yoshida, X. J. Zhou, Z. Hussain, Z.-X. Shen, A. Fujimori, H. Eisaki, and S. Uchida: Phys. Rev. B 80 (2009) 052504.

41) N. Fujiwara, N. Môri, Y. Uwatoko, T. Matsumoto, N. Motoyama, and S. Uchida: Phys. Rev. Lett. 90 (2003) 137001.

42) N. Fujiwara, Y. Fujimaki, S. Uchida, K. Matsubayashi, T. Matsumoto, and Y. Uwatoko: Phys. Rev. B 80 (2009) 100503. 